\title{
СВОЙСТВА ПЛЕНОК СУЛЬФИДА СВИНЦА, ОСАЖДЕННЫХ ИЗ КООРДИНАЦИОННОГО СОЕДИНЕНИЯ $\left[\mathrm{Pb}\left(\mathrm{N}_{2} \mathrm{H}_{4} \mathrm{CS}\right)_{2}\left(\mathrm{CH}_{3} \mathrm{COO}\right)_{2}\right]$
}

\author{
(C) 2017 В. А. Карнушина ${ }^{1}$, В. Н. Семенов ${ }^{1}$, А. Н. Лукин ${ }^{1}$, Н. М. Овечкина ${ }^{2}$, Л. Н. Никитин ${ }^{3}$ \\ ${ }^{1}$ Воронежский государственный университет, Университетская пл., 1, 394018 Воронеж, Россия, \\ e-mail: semenov@chem.vsu.ru \\ ${ }^{2}$ Воронежский государственный медицинский университет им. Н.Н. Бурденко, ул. Студенческая, 10, \\ 394036 Воронеж, Россия \\ ${ }^{3}$ Воронежскийгосударственный техническийуниверситет, Московский пр., 14, 394026 Воронеж, Россия
}

Поступила в редакцию 17.04.2017 г.

\begin{abstract}
Аннотация. Приведены результаты оптической спектроскопии, по которым были исследованы положение, форма края собственного поглощения и определено значение ширины запрещенной зоны тонких пленок сульфида свинца, полученных методом пиролиза аэрозоля водных растворов тиокарбамидных координационных соединений (ТКС). Методом рентгенофазового анализа была определена кристаллическая структура полученных пленок. Методом горячего зонда при комнатной температуре были измерены значения дифференциальной термоэлектродвижущей силы (ТЭДС), удельного сопротивления и удельной электропроводности пленок $\mathrm{PbS}$ при различных соотношениях концентраций исходных компонентов.
\end{abstract}

Ключевые слова: тиокарбамид, ацетат свинца, координационные соединения, комплексообразование, спектры пропускания, рентгенофазовый анализ, ТЭДС, электросопротивление.

\section{ВВЕДЕНИЕ}

В качестве чувствительных элементов полупроводниковых фотосопротивлений широко применяются бинарные сульфидные соединения. Одним из самых перспективных для использования в инфракрасной области является сульфид свинца. В связи с этим большой интерес представляет собой возможность синтеза полупроводниковых пленок $\mathrm{PbS}$ из водных растворов на основе комплексов свинца с тиокарбамидом. Из всех используемых в настоящее время способов получения $\mathrm{PbS}$ наибольший интерес представляет собой метод химического осаждения из растворов [1]. Данный метод позволяет получать тонкие пленки халькогенидов металлов, открывая большие возможности для синтеза новых соединений. Процесс осаждения сульфида свинца методом пиролиза аэрозоля водных растворов ТКС на нагретую подложку протекает через образование промежуточного тиокарбамидного комплекса [2], термическая деструкция которого обуславливает образование сульфида металла [3]. Ранее проведенные исследования [4] показали, что при взаимодействии в водных растворах соли ацетата свинца и тиокарбамида образуется комплекс состава $\left[\mathrm{Pb}\left(\mathrm{N}_{2} \mathrm{H}_{4} \mathrm{CS}\right)_{2}\left(\mathrm{CH}_{3} \mathrm{COO}\right)_{2}\right]$. В связи с этим актуальной задачей являлось изучение процесса осаждения тонких пленок сульфида свинца из водных растворов $\mathrm{Pb}\left(\mathrm{CH}_{3} \mathrm{COO}\right)_{2}$ и $\mathrm{N}_{2} \mathrm{H}_{4} \mathrm{CS}$, a также исследование кристаллической структуры и свойств пленок $\mathrm{PbS}$, синтезируемых в различных условиях [5]. Изучение свойств полученных пленок является основой данной статьи, поскольку они подтверждают правильность выбранного способа их синтеза и устанавливают зависимость свойств от условий получения.

\section{ЭКСПЕРИМЕНТАЛЬНАЯ ЧАСТЬ}

Синтез ТКС проводили путем осаждения из водных растворов ацетата свинца и тиокарбамида в виде осадка следующим образом: были приготовлены шесть растворов, концентрация соли $\mathrm{Pb}\left(\mathrm{CH}_{3} \mathrm{COO}\right)_{2}$ в которых во всех случаях составляла 0.5 моль/л, а содержание $\mathrm{N}_{2} \mathrm{H}_{4} \mathrm{CS}$ по отношению исходных концентраций $\mathrm{C}\left(\mathrm{Pb}\left(\mathrm{CH}_{3} \mathrm{COO}\right)_{2}\right): \mathrm{C}\left(\mathrm{N}_{2} \mathrm{H}_{4} \mathrm{CS}\right)$ составляло $1: 1,1: 2,1: 3,1: 4,1: 5,2: 1[4]$.

Тонкие пленки сульфида свинца были получены методом пиролиза аэрозоля водных растворов $\mathrm{Pb}\left(\mathrm{CH}_{3} \mathrm{COO}\right)_{2}-\mathrm{N}_{2} \mathrm{H}_{4} \mathrm{CS}$ в различных условиях осаждения и варьировании кон- 
центрации тиокарбамида в пределах от 0.1 до 0.5 моль/л при постоянной концентрации ацетата свинца 0.1 моль/л. Полученные образцы $\mathrm{PbS}$ представляли собой плотные пленки темно-серого цвета, не имеющие локальных неоднородностей и обладающие высокой адгезионной способностью по отношению к подложке. Температура подложки $\left(T_{n}\right)$ в процессе синтеза тонких пленок составляла 400 и $450{ }^{\circ} \mathrm{C}$.

Спектры пропускания пленок $\mathrm{PbS}$ были измерены с помощью Фурье-спектрометра Vertex 70. Исследование оптических свойств тонкопленочных структур заключалось в определении значений ширины запрещенной зоны для образцов, синтезированных в различных условиях. Были получены спектры пропускания тонких пленок, с помощью которых по краю собственного поглощения были определены значения ширины запрещенной зоны для прямых переходов путем аппроксимации линейного участка спада коэффициента $(\alpha d)^{1 / n}$ на ось абсцисс энергии фотона $h v$.

Спектры пропускания полученных образцов были получены на Фурье спектрометре Vertex 70 в диапазоне волновых чисел 6000-400 $\mathrm{cm}^{-1}$. С использованием формулы Ламберта-Бугера из экспериментальных спектров была рассчитана зависимость оптической плотности $\alpha d$ от энергии квантов. Графический анализ края собственного поглощения с использованием формулы Тауца [6]: $(\alpha d)^{1 / n}=\left(h v-E_{g}\right)$, где $E_{\mathrm{g}}-$ ширина запрещенной зоны, $h v$ - энергия фотона, $n-$ в зависимости от перехода ( $n=1 / 2$ для прямого перехода, $n=2$ для непрямого перехода) [7], позволил выявить тип оптического перехода и энергию края собственного поглощения тонких пленок сульфида свинца.

Был проведен рентгенофазовый анализ, необходимый для определения фазового состава и структуры сформированных тонких пленок $\mathrm{PbS}$. Анализ образцов, полученных на кварцевых подложках, проводили на рентгеновском дифрактометре PANalytical EMPYREAN. Фазовый состав пленок был выявлен при сравнении полученных из дифрактограмм значений межплоскостных расстояний $d_{h k l}$ с табличными данными, взятыми из справочника[8].

\section{РЕЗУЛЬТАТЫ И ИХ ОБСУЖДЕНИЕ}

Для исследования оптических свойств тонкопленочных образцов сульфида свинца были построены степенные зависимости коэффициента пропускания от энергии фотона и проведена экстраполяция линейных участков функций $(\alpha d)^{2}=f(h \mathrm{n})$ к нулю, что позволило определить значение ширины запрещенной зоны для прямых переходов в различных условиях синтеза.

Учет толщины и зеркального отражения данных образцов представлял некоторую сложность, поэтому для удобства сравнения оптических спектров образцов разного состава нами был использован метод нормализации спектров пропускания по формулам:

$$
\begin{gathered}
T_{\text {нормир }}=e^{-\alpha d}, \alpha d_{\text {нормир }}=\ln \left(\frac{1}{T_{\text {нормир }}}\right), \\
T_{\text {нормир }}=\ln \left(\frac{T_{\max }-T_{\min }}{T(\lambda)-T_{\min }}\right),
\end{gathered}
$$

где $T_{\text {нормир }}-$ нормированное значение пропускания, $T_{\max }$ - максимальное значение пропускания, $T_{\min }-$ минимальное значение пропускания, $T_{(\lambda)}-$ значение пропускания при заданной длине волны $\lambda$.

После чего была получена следующая зависимость:

$$
\alpha d_{\text {нормир }}=\ln \left(\frac{T_{\max }-T_{\min }}{T(\lambda)-T_{\min }}\right) .
$$

Для анализа исследуемых графических зависимостей $T_{\text {нормир }}=f(h v)$ был выбран участок в диапазоне энергий от 0.45 до 0.60 эВ, поскольку в данном промежутке находятся края поглощения, соответствующие прямым межзонным переходам. На рис. 1 представлены края поглощения образцов PbS в выбранном диапазоне энергий:

Как видно на рис. 1, края поглощения расширены из-за мелкой кристалличности синтезируемых образцов, из-за наличия в них рентгеноаморфных фаз и большого количества примесных центров в запрещенной зоне, что является причиной отклонения края поглощения от линейной зависимости, характерной для прямых межзонных переходов.

Соответствующие расшифровки степенных зависимостей $\left(\alpha d_{\text {нормир }}\right)^{2}$ от энергии фотона для образцов при оптимальном соотношении исходных концентраций компонентов $\mathrm{C}\left(\mathrm{Pb}\left(\mathrm{CH}_{3} \mathrm{COO}\right)_{2}\right)$ : $\mathrm{C}\left(\mathrm{N}_{2} \mathrm{H}_{4} \mathrm{CS}\right)=1: 5$ приведены на рис. 2. На графиках детально проиллюстрирована процедура определения значений ширины запрещенной зоны путем экстраполяции линейных участков функций $\left(\alpha d_{\text {нормир }}\right)^{2}=f(h v)$ к нулю.

Анализ функций $\left(\alpha d_{\text {нормир }}\right)^{2}=f(h v)$ показал, что для полученных материалов характерны прямые переходы с оптической шириной запрещенной зоны в диапазоне 0.43-0.49 эВ (табл. 1). 
$a$
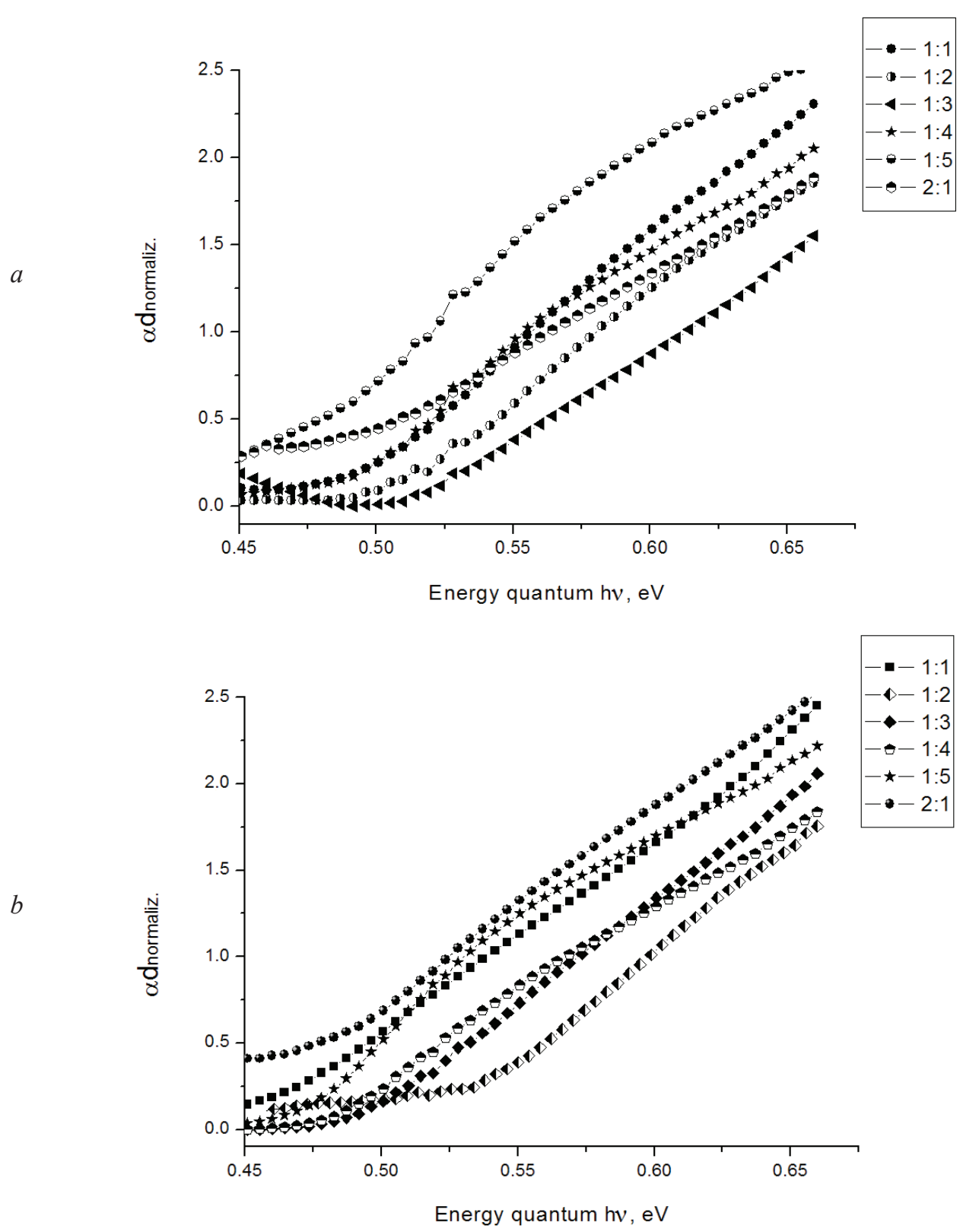

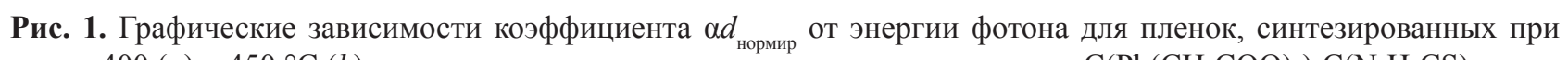
$400(a)$ и $450{ }^{\circ} \mathrm{C}(b)$ и различных соотношениях исходных компонентов $\mathrm{C}\left(\mathrm{Pb}\left(\mathrm{CH}_{3} \mathrm{COO}\right)_{2}\right): \mathrm{C}\left(\mathrm{N}_{2} \mathrm{H}_{4} \mathrm{CS}\right)$

[Fig. 1. The graphical dependences of the coefficient $\alpha d_{\text {normaliz }}$ of the photon energy for films synthesized at $400(a)$ and $450{ }^{\circ} \mathrm{C}(b)$ and different ratios of the initial components $\left.\mathrm{C}\left(\mathrm{Pb}\left(\mathrm{CH}_{3} \mathrm{COO}\right)_{2}\right): \mathrm{C}\left(\mathrm{N}_{2} \mathrm{H}_{4} \mathrm{CS}\right)\right]$

Результаты рентгенофазового анализа пленок $\mathrm{PbS}$, синтезированных при температуре $400{ }^{\circ} \mathrm{C}$ и при соотношении исходных компонентов $C\left(\mathrm{~Pb}\left(\mathrm{CH}_{3} \mathrm{COO}\right)_{2}\right): C\left(\mathrm{~N}_{2} \mathrm{H}_{4} \mathrm{CS}\right)=1: 2$ представлены на рис. 3.

Приведенные данные показали, что при этом соотношении исходных компонентов и температуpe $400{ }^{\circ} \mathrm{C}$ на подложке происходит формирование пленок сульфида свинца кубической модификации, поскольку все линии, характерные для данной модификации, присутствуют на экспериментально по- лученных дифрактограммах, интенсивность этих линий и значения межплоскостных расстояний хорошо сопоставимы с литературными значениями, взятыми из базы данных [8] (табл. 2). Следует отметить, что пленки $\mathrm{PbS}$ осажденные при $400{ }^{\circ} \mathrm{C}$ имеют идентичные значения межплоскостных расстояний в сравнении с литературными данными.

Приведенные в табл. 2 результаты свидетельствуют о том, что в процессе синтеза при всех температурах осаждения в диапазоне $300-450{ }^{\circ} \mathrm{C}$ происходит формирование пленок сульфида свин- 


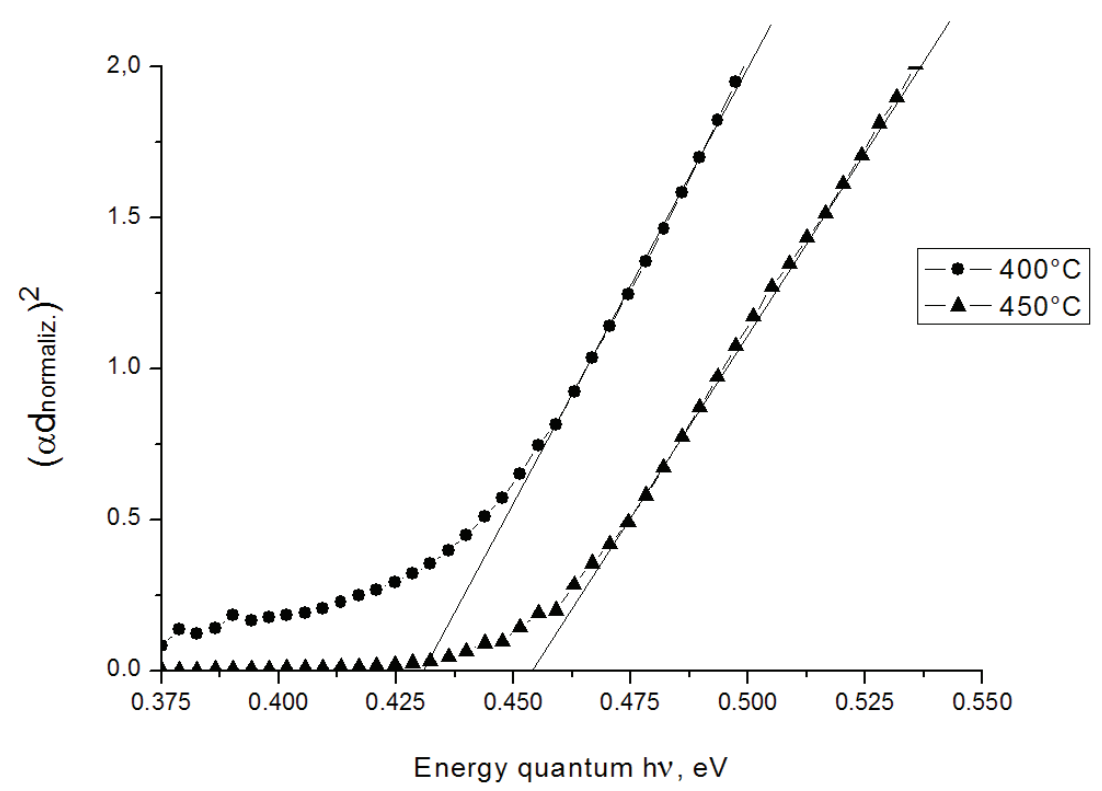

Рис. 2. Степенные зависимости коэффициента $\left(\alpha d_{\text {нормир }}\right)^{2}$ от энергии фотона для пленок, синтезированных при 400 и $450{ }^{\circ} \mathrm{C}$ и соотношении исходных компонентов 1:5

[Fig. 2. Power-law dependencies of the coefficient $\left(\alpha d_{\text {normaliz }}\right)^{2}$ on the photon energy for films synthesized at 400 and $450{ }^{\circ} \mathrm{C}$ and the ratio of the initial components is $1: 5]$

Таблица 1. Значения ширины запрещённой зоны для прямых $E_{g d}$ переходов образцов $\mathrm{PbS}$, синтезированных при температурах 400 и $450{ }^{\circ} \mathrm{C}$

[Table 1. The values of band gap for direct $E_{g d}$ transitions of $\mathrm{PbS}$ samples synthesized at temperatures of 400 and $450 \mathrm{C}$ ]

\begin{tabular}{|c|c|c|}
\hline $\mathrm{C}\left(\mathrm{Pb}\left(\mathrm{CH}_{3} \mathrm{COO}\right)_{2}\right): \mathrm{C}_{\left(\mathrm{N}_{2} \mathrm{H}_{4} \mathrm{CS}\right)}$ & $T_{n},{ }^{\circ} \mathrm{C}$ & $E_{g d},{ }^{\ni B}$ \\
\hline $1: 1$ & 400 & 0.48 \\
\hline $1: 2$ & 400 & 0.49 \\
\hline $1: 3$ & 400 & 0.47 \\
\hline $1: 4$ & 400 & 0.45 \\
\hline $1: 5$ & 400 & 0.43 \\
\hline $2: 1$ & 400 & 0.43 \\
\hline $1: 1$ & 450 & 0.46 \\
\hline $1: 2$ & 450 & 0.48 \\
\hline $1: 3$ & 450 & 0.49 \\
\hline $1: 4$ & 450 & 0.45 \\
\hline $1: 5$ & 450 & 0.43 \\
\hline $2: 1$ & 450 & 0.45 \\
\hline
\end{tabular}

ца кубической структуры с параметром решетки $a=5.9315 \AA$.

Для тонких пленок сульфида свинца были проведены измерения электрического сопротивления $(R)$, дифференциальной ТЭДС, удельного сопротивления ( $\rho)$ и удельной электропроводности $(\sigma)$ методом горячего зонда при комнатной темпера- туре. Результаты данного исследования приведены в табл. 3:

Как видно из табл. 3, с увеличением концентрации тиокарбамида дифференциальная ТЭДС уменьшается, а удельная электропроводность возрастает, что связано с повышением доли комплекса $\left[\mathrm{Pb}\left(\mathrm{N}_{2} \mathrm{H}_{4} \mathrm{CS}\right)_{2}\left(\mathrm{CH}_{3} \mathrm{COO}\right)_{2}\right]$ в исходном растворе за счет координационного взаимодействия $\mathrm{Pb}\left(\mathrm{CH}_{3} \mathrm{COO}\right)_{2} \mathrm{cN}_{2} \mathrm{H}_{4} \mathrm{CS}$. Вследствие этого в слоях $\mathrm{PbS}$ остается меньше примесей, связанных с термодеструкцией. В случае малого значения удельной электропроводности, например, для образца, синтезируемого при соотношении исходных компонентов 1:1, термодеструкция протекала не полностью, в результате чего происходило увеличение количества неконтролируемых примесей и, соответственно, возрастало число примесных состояний в запрещенной зоне.

\section{ВЫВОДЫ}

1. Рентгенофазовый анализ исследуемых пленок $\mathrm{PbS}$ показал, что в процессе синтеза при всех температурах осаждения в диапазоне $300-450{ }^{\circ} \mathrm{C}$ происходит формирование пленок сульфида свинца кубической структуры, о чем свидетельствует присутствие всех характерных линий на полученных дифрактограммах, а также достаточно хорошая сопоставимость значений интенсивностей 


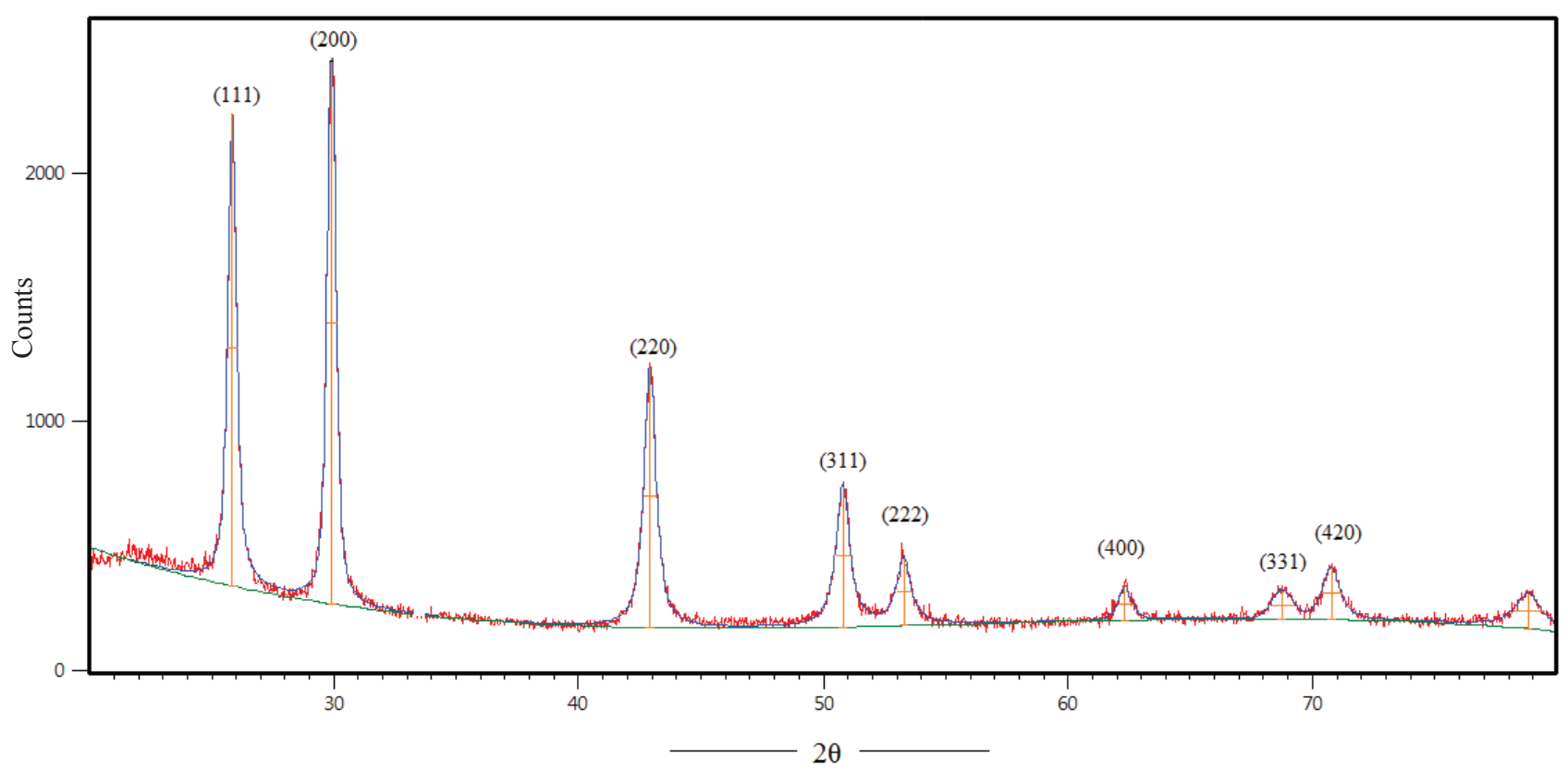

Рис. 3. Дифрактограмма тонкой пленки $\mathrm{PbS}$ при соотношении исходных компонентов $\mathrm{C}\left(\mathrm{Pb}\left(\mathrm{CH}_{3} \mathrm{COO}\right)_{2}\right): C\left(\mathrm{~N}_{2} \mathrm{H}_{4} \mathrm{CS}\right)=1: 2$, синтезированной при $400{ }^{\circ} \mathrm{C}$

[Fig. 3. The X-ray diffraction pattern of a thin film of $\mathrm{PbS}$ at the ratio of the initial components $C\left(\mathrm{~Pb}\left(\mathrm{CH}_{3} \mathrm{COO}\right)_{2}\right): C\left(\mathrm{~N}_{2} \mathrm{H}_{4} \mathrm{CS}\right)=1: 2$, synthesized at $\left.400 \mathrm{C}\right]$

Таблица 2. Сравнительная характеристика межплоскостных расстояний синтезируемых при различных температурах пленок $\mathrm{PbS}$ с литературными значениями

[Table 2. Comparative characteristics of the interplanar spacings for the samples synthesized at different temperatures of films of $\mathrm{PbS}$ with literary values]

\begin{tabular}{|c|c|c|c|c|c|}
\hline \multicolumn{2}{|c|}{$\begin{array}{c}\text { По литературным данным [8] } \\
\text { [Reference [8]] }\end{array}$} & \multicolumn{4}{|c|}{$\begin{array}{c}\text { Экспериментально полученные значения межплоскостных } \\
\text { расстояний пленок } \mathrm{PbS} \text { при разных температурах синтеза, } \AA \\
\text { [The experimental values of the crystal structure of the PbS films } \\
\text { at different synthesis temperatures, } \AA \text { ] }\end{array}$} \\
\hline$h k l$ & $d, \AA$ & $300{ }^{\circ} \mathrm{C}$ & $350^{\circ} \mathrm{C}$ & $400^{\circ} \mathrm{C}$ & $450^{\circ} \mathrm{C}$ \\
\hline (111) & 3.425 & 3.454 & 3.443 & 3.441 & 3.444 \\
\hline (200) & 2.966 & 2.989 & 2.985 & 2.983 & 2.987 \\
\hline$(220)$ & 2.097 & 2.110 & 2.105 & 2.103 & 2.105 \\
\hline (311) & 1.788 & 1.799 & 1.795 & 1.794 & 1.796 \\
\hline (222) & 1.712 & 1.722 & 1.717 & 1.715 & 1.716 \\
\hline$(400)$ & 1.483 & 1.490 & 1.488 & 1.487 & 1.488 \\
\hline (331) & 1.361 & 1.366 & 1.364 & 1.362 & 1.363 \\
\hline$(420)$ & 1.326 & 1.332 & 1.330 & 1.329 & 1.330 \\
\hline
\end{tabular}

Таблица 3. Значения электрического сопротивления, дифференциальной ТЭДС, удельного сопротивления и удельной электропроводности пленок $\mathrm{PbS}$ при различном соотношении концентрации исходных компонентов $\mathrm{Pb}\left(\mathrm{CH}_{3} \mathrm{COO}\right)_{2}$ и $_{2} \mathrm{H}_{4} \mathrm{CS}$

[Table 3. The values of the electrical resistance, the differential thermoelectromotive force, the resistivity and the electrical conductivity of the $\mathrm{PbS}$ films at different ratios of the concentrations of the initial components $\mathrm{Pb}\left(\mathrm{CH}_{3} \mathrm{COO}\right)_{2}$ and $\left.\mathrm{N}_{2} \mathrm{H}_{4} \mathrm{CS}\right]$

\begin{tabular}{|c|c|c|c|c|}
\hline $\begin{array}{c}\mathrm{C}\left(\mathrm{Pb}\left(\mathrm{CH}_{3} \mathrm{COO}\right)_{2}\right): \\
\mathrm{C}\left(\mathrm{N}_{2} \mathrm{H}_{4} \mathrm{CS}\right)\end{array}$ & $R, \mathrm{OM}$ & $\begin{array}{c}\text { ТЭДС, мкВ/К } \\
\text { [differential thermo- } \\
\text { electromotive force }\end{array}$ & $\rho, \mathrm{Oм}^{*} \mathrm{M}$ & $\sigma, 1 / \mathrm{OM}^{*} \mathrm{M}$ \\
\hline $1: 1$ & $9.5 \mathrm{MOM}$ & +907 & 1.900 & 0.526 \\
\hline $1: 2$ & $2 \mathrm{MOM}$ & +613 & 1.000 & 1.000 \\
\hline $1: 3$ & $570 \mathrm{KOM}$ & +547 & 0.175 & 5.702 \\
\hline $1: 4$ & $590 \mathrm{KOM}$ & +507 & 0.148 & 6.779 \\
\hline
\end{tabular}


этих линий с литературными значениями, взятыми из базы данных.

2. Результаты оптических исследований тонкопленочных структур $\mathrm{PbS}$ свидетельствуют о том, что для сульфида свинца характерны прямые переходы с шириной запрещенной зоны от 0.43 до 0.49 эВ. Было выявлено, что значения ширины запрещенной зоны для образцов $\mathrm{PbS}$, синтезированных при соотношении исходных концентраций компонентов 1:5, достаточно хорошо сопоставимы с литературными значениями, поэтому данное соотношение можно считать оптимальным для синтеза тонких пленок сульфида свинца.

3. С увеличением концентрации тиокарбамида в исходном растворе наблюдается уменьшение дифференциальной ТЭДС и увеличение удельной электропроводности в пленках $\mathrm{PbS}$. По нашему мнению, это связано с уменьшением количества примесей в слоях за счет более полного координационного взаимодействия между $\mathrm{Pb}\left(\mathrm{CH}_{3} \mathrm{COO}\right)_{2}$ и $\mathrm{N}_{2} \mathrm{H}_{4} \mathrm{CS}$.
Работа выполнена с привлечением приборного парка ЦКПНО ВГУ.

\section{СПИСОК ЛИТЕРАТУРЫ}

1. Марков В. Ф., Маскаева Л. Н., Иванов П. Н. Гидрохимический синтез пленок сульфидов металлов: моделирование и эксперимент. Екатеринбург, УрО РАН, 2006, $218 \mathrm{c}$.

2. Наумов А. В., Семенов В. Н., Авербах Е. М. // Хuмическая промышленность, 2003, т. 80, № 2, с. 17-26.

3. Семенов В. Н., Наумов А. В. // Журнал общей химии, 2001, т. 27, № 4, 533 с.

4. Семенов В. Н., Карнушина В. А., Овечкина Н. М. // Вестник ВГУ, серия: Химия. Биология. Фармаичи, 2016, № 1 , с. $25-29$.

5. Fedjukin A. V., Nituta A. N., Lukin A. N., Semenov V. N. // Bulletin of the Russian Academy of Sciences. Physics, 2015, № 2, p. 79.

6. Уханов Ю. И. Оптические свойства полупроводников. М.: Наука, 1977, 368 с.

7. Ахмедов О. Р., Гусейналиев М. Г., Абдуллаев Н. А. // ФТП, 2016, т. 50, № 1, с. 51-54.

8. Миркин Л. И. Справочник по рентгеноструктурному анализу поликристаллов. М.: Физматгиз, 1961, 863 с.

\title{
PROPERTIES OF LEAD SULFIDE FILMS DEPOSITED FROM A COORDINATION COMPOUND $\left[\mathrm{Pb}\left(\mathrm{N}_{2} \mathrm{H}_{4} \mathrm{CS}\right)_{2}\left(\mathrm{CH}_{3} \mathrm{COO}\right)_{2}\right]$
}

\author{
(C) 2017 V. A. Karnushina ${ }^{1}$, V. N. Semenov ${ }^{1}$, A. N. Lukin ${ }^{1}$, N. M. Ovechkina ${ }^{2}$, L. N. Nikitin ${ }^{3}$ \\ ${ }^{1}$ Voronezh State University, 1 Universitetskaya sq., 394018 Voronezh, Russia \\ e-mail:semenov@chem.vsu.ru \\ ${ }^{2}$ Burdenko Voronezh State Medical University, Voronezh Base University, 10 Studencheskaya str., \\ 394036 Voronezh, Russia \\ ${ }^{3}$ Voronezh State Technical University, 14 Moscovsky av., 394026 Voronezh, Russia
}

Received 17.04.2017

\begin{abstract}
The position and the shape of intrinsic absorption edges was studied by optical spectroscopy. It allowed identifying the value of the band gap for thin lead sulfide films produced by spray pyrolysis of aqueous solutions of thiourea coordination compounds (TCS). The study of the optical properties of $\mathrm{PbS}$ was focsued on determining the values of the band gap for samples which were synthesized in various conditions. The transmission spectra for thin films were determined, which helped to determine the band gap for direct interband transitions by the intrinsic self-absorption edge. Graphical analysis of the intrinsic absorption edge allowed us to identify the type of optical transition and the energy of the intrinsic absorption edge for thin films of lead sulfide. By X-ray phase analysis the crystal structure of the films was determined. Using the hot probe method at room temperature, we measured the values of electric resistance, differential thermoelectric power, and the resistivity and conductivity of $\mathrm{PbS}$ films with different ratios of concentrations of the initial components. The results of X-ray phase analysis showed that films of lead sulfide with cubic structure are formed as a result of synthesis at all deposition temperatures between 300-450 C. The results of optical studies of PbS thin film structures indicate that lead sulfide is characterized by direct transitions with a band gap within 0.43 to $0.49 \mathrm{eV}$. The increase in concentration of thiourea in the initial solution leads to the decrease in the differential thermoelectric power and the increase in conductivity in $\mathrm{PbS}$ films.
\end{abstract}


Keywords: thiourea, lead acetate, coordination compounds, complexing, transmission spectra, X-ray phase analysis, TEPS, electrical resistivity.

\section{ACKNOWLEDGEMENTS}

The research results were obtained with equipment of Voronezh State University Centre for Collective Use of Scientific Equipment

\section{REFERENCES}

1. Markov V. F., Maskaeva L. N., Ivanov P. N. Hydrochemical Synthesis of Films of Metal Sulphides: Modeling and Experiment. Ekaterinburg, UrB RAS Publ., 2006, 218 p. (in Russian)

2. Naumov A. V., Semenov V. N., Averbakh E. M. Chemical Industry, 2003, vol. 80, no. 2, pp. 17-26. (in Russian)

3. Semenov V. N., Naumov A. V. J. of General Chemistry, 2001, vol. 27, no. 4, 533 p. (in Russian)
4. Semenov V. N., Karnushina V. A., Ovechkina N. M. Bulletin of VSU, series: Chemistry. Biology. Pharmacy, 2016, no. 1, p. 25-29. Available at: http://www.vestnik.vsu. ru/pdf/chembio/2016/01/2016-01-05.pdf (in Russian)

5. Fedjukin A. V., Nituta A. N., Lukin A. N., Semenov V. N. Bulletin of the Russian Academy of Sciences. Physics, 2015, no. 2, p. 79. DOI: 10.3103/S1062873815020 100 Available at: http://elibrary.ru

6. Ukhanov Yu. I. Optical Properties of Semiconductors. Moscow, Science Publ., 1977, 368 p. (in Russian)

7. Akhmedov O. R., Guseinaliev M. G., Abdullaev N. A. Physics and Technology of Semiconductors, 2016, vol. 50, no. 1, pp. 51-54.DOI: 10.1134/S1063782616010036 Available at: http://elibrary.ru

8. Mirkin L. I. Handbook of X-ray Diffraction Analysis of Polycrystals. Moscow, Fizmatgiz Publ., 1961, 863 p.(in Russian)
Карнушина Виктория Александровна - магистрант кафедры общей и неорганической химии, Воронежский государственный университет; тел.: +7(980) 3416041, e-mail: 79803416041@yandex.ru

Семенов Виктор Николаевич - д. х. Н., профессор, заведующий кафедрой общей и неорганической химии, Воронежский государственный университет; тел.: +7(473) 2208610, e-mail: semenov@chem.vsu.ru

Лукин Анатолий Николаевич - к. ф.-м. н., доцент кафедры физики твердого тела и наноструктур, Воронежский государственный университет; тел.: +7(950) 7548707, e-mail:ckp_49@mail.ru

Овечкина Надежда Митрофановна - ассистент кафедры химии, Воронежский государственный медицинский университет им. Н. Н. Бурденко; тел. +7(473) 2531479, e-mail: nadezhda.ovechkina@rambler.ru

Никитин Леонид Николаевич - к. т. н., доцент кафедры конструирования и производства радиоаппаратуры, Воронежский государственный технический университет; тел.: +7(473) 2437706, e-mail:1.n.nikitin@ mail.ru
Karnushina Victoria Al. - the competitor for science degree of Master Science in Chemistryof the Department of General and Inorganic Chemistry, Voronezh State University; ph.: +7(980) 3416041, e-mail: 79803416041@ yandex.ru

Semenov Viktor N. - Dr. Sci. (Chem.), Head of the Department of General and Inorganic Chemistry, Voronezh State University; ph.: +7(473) 2208610, e-mail: semenov@ chem.vsu.ru

Lukin Anatoly N. - Cand. Sci. (Phys.-Math.), Associate Professor of the Department of Solid State Physics and Nanostructures, Voronezh State University; ph.: +7(950) 7548707, e-mail:ckp_49@mail.ru

Ovechkina Nadezhda M. - Assistant of the Department of Chemistry, Voronezh State Medical University. N. N. Burdenko; ph.:+7(473) 2531479, e-mail: nadezhda.ovechkina@ rambler.ru

Nikitin Leonid N. - Cand. Sci. (Eng.), Assistant Professor of the Department of Design and Production of Radio Equipment, Voronezh State Technical University; ph.: +7(473) 2437706, e-mail: 1.n.nikitin@mail.ru 